\title{
An anti-diffusive scheme for viability problems
}

\author{
Olivier BOKANOWSKI*, Sophie MARTIN ${ }^{\dagger}$, Remi MUNOS $^{\ddagger}$, HASNAA ZIDANI $^{\S}$
}

\begin{abstract}
This paper is concerned with the numerical approximation of viability kernels. The method described here provides an alternative approach to the usual viability algorithm. We first consider a characterization of the viability kernel as the value function of a related optimal control problem, and then use a specially relevant numerical scheme for its approximation. Since this value function is discontinuous, usual discretization schemes (such as finite differences) would provide a poor approximation quality because of numerical diffusion. Hence, we investigate the Ultra-Bee scheme, particularly interesting here for its anti-diffusive property in the transport of discontinuous functions. Although currently there is no available convergence proof for this scheme, we observed that numerically, the experiments done on several benchmark problems for computing viability kernels and capture basins are very encouraging compared to the viability algorithm, which fully illustrates the relevance of this scheme for numerical approximation of viability problems.
\end{abstract}

Key-words: Viability kernel, Capture basin, Ultra-Bee scheme, Optimal control.

\section{Introduction}

We consider a control system, defined by the differential equation:

$$
\begin{aligned}
& \dot{y}(t)=f(y(t), u(t)), \quad u(t) \in U \text { for almost every } t \geq 0 \\
& y(0)=x_{0}
\end{aligned}
$$

where $f: \mathbb{R}^{n} \times \mathbb{R}^{p} \rightarrow \mathbb{R}^{n}$ and $U$ is a compact subset in $\mathbb{R}^{p}$. We denote by $F$ the set-valued map defined by $F(y):=\{f(y, u), u \in U\}$, for every $y \in \mathbb{R}^{n}$.

*Lab. Jacques-Louis Lions, Université Pierre et Marie Curie, 175 Rue Chevaleret 75013 Paris, France. Email: boka@math.jussieu.fr

${ }^{\dagger}$ Lab. d'ingénierie des systèmes complexes, Cemagref, 24 av. des Landais, BP 50085, 63172 Aubière Cedex, France. Email: sophie.martin@clermont.cemagref.fr

$\ddagger$ Centre de Mathmatiques Appliques, Ecole Polytechnique, 91128 Palaiseau Cedex, France

${ }^{\S}$ ENSTA, UMA, 32 Boulevard Victor, 75739 Paris Cedex 15, France. Email: Hasnaa.Zidani@ensta.fr. Also at Projet Sydoco, INRIA Rocquencourt, BP. 105, 78153 Le Chesnay Cedex. 
Following Aubin [2], we say that a solution $y(\cdot)$ of $(1)$ is viable in a constrained set $K \subset \mathbb{R}^{n}$, under the dynamics $F$, if it remains in $K$ forever:

$$
\forall t \geq 0, y(t) \in K
$$

The Viability Kernel of $K$ under $F$, denoted by $\operatorname{Viab}_{F}(K)$, is defined as the set of points $x_{0}$ from which can start a viable solution, i.e.,

$$
\begin{aligned}
\operatorname{Viab}_{F}(K):= & \left\{x_{0} \in K, \exists u \in L^{\infty}((0,+\infty) ; U)\right. \\
& \text { the solution of }(1) \text { associated to } u \text { satisfies } \forall t \geq 0, y(t) \in K\} .
\end{aligned}
$$

The viability theory aims at controlling dynamical systems with the goal to maintain them inside a given constrained set. Such problems are frequent in ecology or economics, where the systems die or badly deteriorate when they leave some regions of the state space. For instance Béné et al. studied the management of a renewable resource as a viability problem [6]. They pointed out irreversible overexploitation configurations related to the resource extinction. Mullon et al. proposed a viability model of the trophic interactions in marine ecosystems [18]. Bonneuil studied the conditions the prey-predator dynamics must satisfy to avoid the extension of one or the other species as a viability problem [8]. Recent applications of viability concepts can also be found in finance (Saint-Pierre [22]) and in the evaluation of the resilience of socio-ecological systems (Martin [17]).

The viability kernel may be characterized in diverse ways through tangential conditions thanks to the viability theorems [3].

The viability kernel algorithm, proposed by Saint-Pierre [20] computes for a given grid $G_{h}$, a discrete viability kernel that converges to the viability kernel $\operatorname{Viab}_{F}(K)$ when the grid resolution $h$ tends to 0 .

In this paper, we investigate an alternative approach. First, we characterize the viability kernel by the infinite time limit of the value function of an evolutionary control problem.

This value function being discontinuous, usual discretization schemes such as those based on interpolation techniques (like Semi-Lagrangian or finite differences methods developped for continuous value functions [10, 1]) fail to provide accurate approximations because of numerical diffusion [13].

Here, we propose to use the anti-diffusive Ultra-Bee scheme extended to the resolution of Hamilton-Jacobi-Bellman equations [7], which we believe is particularly relevant to the specific shape of the value functions derived from viability problems.

So far, no convergence proof for this scheme is available. However, the numerical experiments tested on several benchmark problems and compared to the viability algorithm are very encouraging, in terms of the approximation error.

Extension to the computation of the capture basin of a target $C \subset K$ (defined as the set of initial states $x_{0} \in K$ such that $C$ is reached in finite time before possibly leaving $K$ by at least one trajectory $y(\cdot))$ is also treated and illustrated. 
The paper is organized as follows. In section 2, we define value functions related to the above problems, and give Hamilton-Jacobi-Bellman (HJB) equations satisfied by these value functions. In section 3, we recall the Ultra-Bee scheme adapted to treat HJB equations, and also define an Ultra-Bee scheme for computing a capture basin. In section 4 we compare on various examples the numerical results given by the Ultra-Bee scheme and by the viability algorithm (we deeply thank P. Saint-Pierre for allowing us to use his code).

For sake of completeness we also recall the viability algorithm in the appendix.

\section{Statement of the problem and basic results}

Let $K$ be a non empty compact subset of $\mathbb{R}^{n}$, and let $U$ be a non empty compact convex subset of $\mathbb{R}^{p}$. Let $f: \mathbb{R}^{n} \times U \longrightarrow \mathbb{R}^{n}$ satisfies:

(A1) $f$ is a continuous function. There exists two functions $g(x)$ and $h(x)$ such that $f(x, u)=g(x)+h(x) \cdot u$. There exists $c_{o} \geq 0$ s.t. $\sup _{u \in U}|f(x, u)| \leq c_{o}(1+$ $|x|)$. Moreover, for every $R>0$, there exists $L_{R}>0$, such that $\forall x, y \in$ $B(0, R), \sup _{u \in U}|f(x, u)-f(y, u)| \leq L_{R}|x-y|$.

Remark 2.1. Note that in what follows, it could be also possible to consider more general functions $f$, replacing the assumption $f(x, u)=g(x)+h(x) \cdot u$ by for instance the fact that for all $x$, the subset $\{f(x, u), u \in U\}$ is convex and compact.

\subsection{Preliminaries and notations}

For any measurable control $u \in \mathcal{U}:=L^{\infty}((0, \infty) ; U)$, we denote by $y_{x}(\cdot)$ the unique absolutely continuous solution of

$$
\dot{y}_{x}(t)=f\left(y_{x}(t), u(t)\right) \quad \text { for a.e. } t \geq 0, \quad y_{x}(0)=x \text {. }
$$

We define the set-valued map $F: \mathbb{R}^{n} \rightarrow \mathbb{R}$ by

$$
F(z):=\{f(z, u) ; u \in U\} .
$$

If (A1) is fulfilled then $F$ is a continuous set-valued map which satisfies a linear growth (i.e. $\exists C \geq 0, \forall x \in \mathbb{R}^{n}$, $\left.\sup _{y \in F(x)}|y| \leq C(1+|x|)\right)$, and has convex compact values. It is easy to check that if $y_{x}$ is a solution of (4), then $y_{x}$ satisfies the differential inclusion:

$$
\dot{z}(t) \in F(z(t)) \quad \text { for a.e. } t \geq 0, \quad z(0)=x .
$$

Conversely, $z$ is a solution of (5) if there exists $u \in L^{\infty}((0, \infty) ; U)$ such that $z$ is the solution of (4) associated to $u[2,3]$. 
In order to deal with capture basin problems in Section 2.3, we shall need to define modified dynamics $F_{C}$, associated to a given compact set $C \subset \mathbb{R}^{n}$, by:

$$
F_{C}(z):= \begin{cases}F(z) & \text { if } z \notin C \\ \operatorname{Conv}\{0, F(z)\} & \text { if } z \in \partial C \\ \{0\} & \text { if } z \in \operatorname{int}(C)\end{cases}
$$

where $\operatorname{int}(C)$ is the interior of $C, \partial C$ is the border of $C$, and $\operatorname{Conv}\{0, F(z)\}$ is the convex hull of $\{0\} \cup F(z)$. Here, under the assumption (A1), the set-valued function $F_{C}$ is upper semi-continuous, ${ }^{1}$ satisfies a linear growth, and has convex compact values. In this case also, the following differential inclusion:

$$
\dot{y}(t) \in F_{C}(y(t)) \quad \text { for a.e. } t \geq 0, \quad y(0)=x
$$

admits absolutely continuous solutions (see [2]). Moreover, for any solution $y$ of (7), there exists $u \in L^{\infty}((0, \infty) ; U)$ and $w \in L^{\infty}((0,+\infty) ;[0,1])$ such that:

$$
\begin{aligned}
& \dot{y}(t)=w(t) f(y(t), u(t)) \quad \text { for a.e. } t \geq 0, \quad y(0)=x, \\
& \text { and } w(t) \in \mathcal{W}(y(t)), \text { for a.e. } t \geq 0
\end{aligned}
$$

where $\mathcal{W}$ is the set-valued map defined by

$$
\mathcal{W}(z)= \begin{cases}\{1\} & \text { if } z \notin C, \\ {[0,1]} & \text { if } z \in \partial C, \quad z \in \mathbb{R}^{n} \\ \{0\} & \text { if } z \in \operatorname{int} C .\end{cases}
$$

(for this fact one can adapt the proof of [2, Chap. 1.14, Corollary 1]).

Remark 2.2. The trajectories of (7) correspond to the same trajectories as the solutions of (4) as long as it evolves outside the set $C$. When a trajectory of (7) touches the border $\partial C$ of $C$, then it may either stay on the border or keep moving under the dynamics $F$. When it starts from a point of the interior of $C$, it doesn't move anymore.

\subsection{Viability kernel}

To determine the viability kernel of $K$ under the dynamics $F$, we define the function

$$
\mathcal{V}(x):= \begin{cases}0 & \text { if } \exists u \in \mathcal{U}, \text { s.t. the solution } y_{x} \text { of }(4) \text { satisfies } y_{x}(t) \in K, \forall t \geq 0 \\ +\infty & \text { otherwise }\end{cases}
$$

Of course we have that $\mathcal{V}(x)=+\infty$ if $x \notin K$. We can also write $\mathcal{V}(x)$ as an optimization problem:

$$
\mathcal{V}(x)=\min \left\{0 \mid \exists u \in \mathcal{U}, y_{x} \text { solution of (4) satisfies } y_{x}(t) \in K, \forall t \geq 0\right\},
$$

\footnotetext{
${ }^{1}$ For any $x_{0}$, for any open $N$ such that $F\left(x_{0}\right) \subset N, \exists M$ neighborhood of $x_{0}, F_{C}(M) \subset N$.
} 
with the convention that $\mathcal{V}(x)=+\infty$ if the set of constraints is empty.

Then the viability kernel is given by

$$
\operatorname{Viab}_{F}(K)=\left\{x \in \mathbb{R}^{n}, \mathcal{V}(x)=0\right\} .
$$

For each $T \geq 0$, we introduce now the following time-dependent value function

$$
V(T, x)= \begin{cases}0 & \text { if } \exists u \in \mathcal{U}, y_{x} \text { solution of }(4), y_{x}(t) \in K \forall t \in[0, T], \\ +\infty & \text { otherwise. }\end{cases}
$$

Moreover, we have

Lemma 2.1. Under assumption (A1), for every $x \in K, V(T, x)$ converges towards $\mathcal{V}(x)$ as $T \rightarrow+\infty$.

Proof. We first remark that, for every $x \in \mathbb{R}^{n}, 0 \leq T \leq T^{\prime}$, we have $V(T, x) \leq V\left(T^{\prime}, x\right)$. Indeed, suppose that $V\left(T^{\prime}, x\right)=0$. Then there exists a control $u$ such that $y_{x}(t) \in K$ for every $t \in\left[0, T^{\prime}\right]$, and in particular for every $t \in[0, T]$. Hence $V(T, x)=0$. Otherwise, in the case $V\left(T^{\prime}, x\right)=+\infty$, the inequality $V(T, x) \leq V\left(T^{\prime}, x\right)$ is also satisfied.

Now, in the case $\mathcal{V}(x)=0$, there exists $u \in \mathcal{U}$, such that the trajectory $y_{x}$ associated to $u$ and starting from $x$ satisfies $y_{x}(t) \in K \forall t \geq 0$. This implies that $V(T, x)=0$ for all $T>0$, and we have $V(T, x) \stackrel{T \rightarrow+\infty}{\rightarrow} 0$.

For the reciprocal, we follow the arguments of [20, theorem 3.1]. Let $x \in K$ such that $\lim _{T \rightarrow+\infty} V(T, x)=0$. This means that for every $n \geq 0$, there exists a trajectory $y_{n}$ solution of $(5)$ (with the initial condition $y_{n}(0)=x$ ) such that $y_{n}(t)$ belongs to $K$ for every $t \in[0, n]$. Using (A1), we obtain the bounds:

$$
\begin{aligned}
& \left|y_{n}(t)\right| \leq(1+|x|) e^{c_{o} t} \quad t \geq 0 \\
& \left|\dot{y}_{n}(t)\right| \leq c_{o}(1+|x|) e^{c_{o} t} \quad \text { a.e. } t>0 ;
\end{aligned}
$$

here $c_{o}$ is the same as in (A1).

Then, the sequence $\left(y_{n}\right)$ is bounded in $W^{1,1}\left(0,+\infty ; e^{-\left(c_{o}+1\right) t} d t\right)$ (which is the space of functions $z \in L^{1}\left(0,+\infty ; e^{-\left(c_{o}+1\right) t} d t\right)$ integrable for the measure $e^{-\left(c_{o}+1\right) t} d t$ and such that there exists a weak derivative $\dot{z} \in L^{1}\left(0,+\infty ; e^{-\left(c_{o}+1\right) t} d t\right)$. By the Ascoli-Alaoglu theorem, we derive that there exists $y \in W^{1,1}\left(0,+\infty ; e^{-\left(c_{o}+1\right) t} d t\right)$ and a subsequence, still denoted by $y_{n}$, such that

$$
\begin{aligned}
& y_{n} \text { converges uniformly to } y \\
& \dot{y}_{n} \text { converges weakly to } \dot{y} \text { in } L^{1}\left(0,+\infty ; e^{-\left(c_{o}+1\right) t} d t\right) .
\end{aligned}
$$

Moreover, by the convergence theorem [2, Chap. 1.4, Th.1], $y$ satisfies

$$
\dot{y}(t) \in F(y(t)) \quad \text { a.e. } t>0, \quad y(0)=x .
$$

On the other hand, for every $t \geq 0$ :

$$
y(t)=\lim _{\substack{n \rightarrow \infty \\ n \geq t}} y_{n}(t) \in K .
$$


We conclude that there exists a trajectory starting at $x$ and lying in $K$ forever, thus $\mathcal{V}(x)=0$ which ends the proof.

Note that this result is not new; a similar result may be found in [3, Chap. 4].

We may also deduce the following result (which proof is left to the reader):

Lemma 2.2. Let $\Omega_{T}:=\{x, V(T, x)=0\}$. Under the assumption (A1), we have

(i) $\Omega_{T^{\prime}} \subset \Omega_{T} \subset K$, for every $T^{\prime} \geq T \geq 0$,

(ii) $\bigcap_{T>0} \Omega_{T}=\operatorname{Viab}_{F}(K)$.

Now we propose to compute $\Omega_{T}$ for $T$ large enough, and to approximate $\operatorname{Viab}_{F}(K)$ by $\Omega_{T}$. We thus look for an approximation for $V(T,$.$) in K$. It is clear that $V$ can be interpreted as a value function of an optimal control problem:

$$
V(T, x)=\min \left\{0 \mid \exists u \in \mathcal{U}, y_{x} \text { solution of }(4) \text { and } y_{x}(t) \in K, \forall t \in[0, T]\right\}
$$

Then $V$ satisfies an Hamilton-Jacobi-Bellman equation of the following form:

$$
\begin{aligned}
& V_{t}-\min _{u \in U}\left(f(x, u) \cdot \nabla_{x} V\right)=0, \quad t>0, x \in K \\
& V(0, x)=0, \quad x \in K .
\end{aligned}
$$

Many works deal with the sense of solution of the above equation. Let us just mention that under hypotheses which include the "inward-pointing" constraint qualification

$$
\min _{u \in U} f(x, u) \cdot \eta_{x}<0 \quad \forall x \in \partial K,
$$

(where $\eta_{x}$ is the unit outward normal vector, in the case $\partial K$ is sufficiently smooth) the value function is continuous and is the unique viscosity solution of (10). The hypothesis (11) was introduced by Soner [23], see also Capuzzo-Dolcetta and Lions [11], Ishii and Koike [15]. In our case, the inward qualification assumption does not necessarily hold, since it would imply that $\operatorname{Viab}_{F}(K)=K$ (in this case, it obvious that $V(T, x)=0$ if $x \in K$ and $V(T, x)=+\infty$ otherwise).

When the "inward-pointing" constraint fails, the characterization of the value function as the lower semi-continuous solution to (10) is provided by Frankowska and Plaskacz [14] under the "outward-pointing" constraint qualification:

$$
\max _{u \in U} f(x, u) \cdot \eta_{x}>0 \quad \forall x \in \partial K .
$$

(In this case, the solution of (10) is no more necessarily $V(T, x)=0$ if $x \in K$ and $V(T, x)=+\infty$ otherwise.) The notion of lower semi-continuous solutions has first been introduced by Barron and Jensen [4, 5]. The results of [14] can been viewed as a generalization of the work of Barron-Jensen done for state constraint-free problems.

These hypotheses (11) or (12) under which the value function may correctly be characterized, will not be further investigated here. Our purpose in this paper is not to provide a theoretical framework for analyzing the solution to this kind of HJB equations, but rather to illustrate the numerical approximation of the value function by using a specific (anti-diffusive) scheme. 


\subsection{Capture basin}

Let us introduce a "target" $C$, a closed subset of $K$. The subset of initial states $x \in K$ such that $C$ is reached in finite time before possibly leaving $K$ by at least one trajectory $y_{x}(\cdot)$ is called the capture basin of $C$ in $K$ and denoted $\operatorname{Capt}_{F}(C)$ :

$$
\begin{aligned}
\operatorname{Capt}_{F}(C):= & \left\{x \in K ; \text { s.t. there exist } y_{x} \text { solution of }(5) \text { and } t \geq 0,\right. \\
& \text { such that } \left.y_{x}(t) \in C, y_{x}(s) \in K, \forall s \in[0, t]\right\} .
\end{aligned}
$$

We now consider the modified dynamics $F_{C}$ defined in (6). It is know that we have $\operatorname{Viab}_{F_{C}}(K)=\operatorname{Capt}_{F}(C) \cup \operatorname{Viab}_{F}(K)$ (see for instance [21]). Hence if $K$ is a "repeller" for $F$, i.e. $\operatorname{Viab}_{F}(K)=\emptyset$, then we have $\operatorname{Capt}_{F}(C)=\operatorname{Viab}_{F_{C}}(K)$. However in general $\operatorname{Viab}_{F}(K) \neq \emptyset$, and we need an other approach for the approximation of $\operatorname{Capt}_{F}(C)$.

For our purpose, let $T>0$. Let $\chi_{C}$ be defined by $\chi_{C}(x):=0$ if $x \in C$ and $\chi_{C}(x)=+\infty$ otherwise. Consider $\vartheta_{T}(\tau, x)$ be defined by

$\vartheta_{T}(\tau, x)=\min _{t \in[\tau, T]}\left\{\chi_{C}\left(y_{\tau, x}(t)\right) ; y_{\tau, x}\right.$ a solution of $(15)$ and $y_{\tau, x}(s) \in K$ for $\left.s \in[\tau, t],\right\}$

where

$$
\dot{y}_{\tau, x}(s) \in F\left(y_{\tau, x}(s)\right) \quad \text { for a.e. } s \geq \tau, \quad y_{\tau, x}(\tau)=x .
$$

(As already mentioned in section 2.1,y is a solution of (15) if and only if there exists a control $u \in \mathcal{U}$ such that $\dot{y}(t)=f(y(t), u(t)), \quad y(\tau)=x$.)

The function $\vartheta_{T}$ takes the value 0 whenever there exists a trajectory $y_{\tau, x}$ solution of (15) which reaches the target $C$ before time $T$ (while staying in $K$ ), otherwise its value is $+\infty$.

Now we define the capture basin before time $T$ by

$$
\operatorname{Capt}_{F}(C ; T):=\left\{x \in K, \quad \vartheta_{T}(0, x)=0\right\} .
$$

In particular, $T \rightarrow \operatorname{Capt}_{F}(C ; T)$ is increasing for the inclusion, and also we find the usual capture basin as $T \rightarrow \infty$ :

$$
\lim _{T \rightarrow \infty} \operatorname{Capt}_{F}(C ; T)=\operatorname{Capt}_{F}(C) .
$$

On the other hand, we remark the following identity (under the assumption (A1)):

$$
\begin{aligned}
& \vartheta_{T}(\tau, x)=\min \left\{\chi_{C}\left(\widetilde{y}_{\tau, x}(T)\right) ;\right. \\
& \left.\widetilde{y}_{\tau, x} \text { solution of }(17) \text { and } \widetilde{y}_{\tau, x}(s) \in K \text { for all } s \in[\tau, T]\right\}
\end{aligned}
$$

where

$$
\dot{\tilde{y}}_{\tau, x}(s) \in F_{C}\left(\widetilde{y}_{\tau, x}(s)\right), \widetilde{y}_{\tau, x}(\tau)=x
$$


(the proof is left to the reader). In this formula the trajectories $\widetilde{y}_{\tau, x}(t)$ are watched up to time $T$, but when entering into the target, they do not move anymore.

Thus the minimal time problem (14) is turned into a Rendez-Vous problem (16) with state constraints. In fact it is not important to know the minimal time for reaching the target, but only to know if the target is reachable in finite time. Note that in the new equivalent problem (16), we can use the expression mentioned in (8a) (and Remark 2.2) to understand the dynamics of the solutions of (7).

Using (16) we can state a dynamic programming principle for $\vartheta_{T}($ for $\tau+\Delta t \leq T)$ :

$$
\begin{aligned}
& \vartheta_{T}(\tau, x)=\min \left\{\vartheta_{T}\left(\tau+\Delta t, \widetilde{y}_{\tau, x}(\tau+\Delta t)\right) ;\right. \\
& \left.\widetilde{y}_{\tau, x}(s) \text { solution de }(17), \widetilde{y}_{\tau, x}(s) \in K \forall s \in[\tau, \tau+\Delta t]\right\} \\
& \vartheta_{T}(T, x)=\chi_{C}(x),
\end{aligned}
$$

Under some technical assumptions, $\vartheta_{T}$ is the lower semi-continuous solution of an HJB equation (see [14]).

\section{Ultra-Bee scheme}

We shall present the Ultra-Bee (UB) scheme for equation (18) in space dimension 2, using three steps: we first present the UB scheme for linear advection in $1 \mathrm{~d}$, then in $2 \mathrm{~d}$, and finally in $2 \mathrm{~d}$ for equation (18).

$U B$ scheme for $1 d$ linear advection. We consider the discretization of

$$
\left\{\begin{array}{l}
v_{t}+f(x) v_{x}=0, \quad t>0, x \in \mathbb{R} \\
v(0, x)=v_{0}(x)
\end{array}\right.
$$

where $x \rightarrow f(x)$ is Lipschitz-continuous, and the initial condition $v_{0}$ is assumed in $L_{\text {loc }}^{1}(\mathbb{R})$. Let $\left(x_{j}\right)$ such that $x_{j+1}-x_{j}=\Delta x$ and $t_{n}=n \Delta t$ be uniform space and time discretizations, where $\Delta x, \Delta t$ are the mesh sizes. Let $V_{j}^{n}$ denotes a numerical

approximation of the solution $v\left(t_{n}, x_{j}\right)$, The UB scheme for (19) takes the following form:

$$
\frac{V_{j}^{n+1}-V_{j}^{n}}{\Delta t}+f\left(x_{j}\right) \frac{V_{j+\frac{1}{2}}^{n, L}-V_{j-\frac{1}{2}}^{n, R}}{\Delta x}=0,
$$

with the initialization:

$$
V_{j}^{0}:=\frac{1}{\Delta x} \int_{x_{j-\frac{1}{2}}}^{x_{j+\frac{1}{2}}} v_{0}(x) d x
$$


where $x_{j+\frac{1}{2}}=x_{j}+\frac{\Delta x}{2}$. Here $V_{j+\frac{1}{2}}^{n, L}$ and $V_{j+\frac{1}{2}}^{n, R}$ are numerical fluxes that will be defined below. We write (20) in the equivalent non-conservative form:

$$
V_{j}^{n+1}=V_{j}^{n}-\nu_{j}\left(V_{j+\frac{1}{2}}^{n, L}-V_{j-\frac{1}{2}}^{n, R}\right),
$$

where

$$
\nu_{j}:=\frac{\Delta t}{\Delta x} f\left(x_{j}\right)
$$

is a "local CFL" number. We assume that $\left|\nu_{j}\right| \leq 1 \forall j$. In the case $\nu_{j}=0$, we thus consider $V_{j}^{n+1}=V_{j}^{n}$, and the fluxes $V_{j+\frac{1}{2}}^{n, R / L}$ need not be defined.

We first set

$$
\begin{aligned}
& \text { if } \nu_{j}>0, \quad\left\{\begin{array}{l}
b_{j}^{+}:=\max \left(V_{j}^{n}, V_{j-1}^{n}\right)+\frac{1}{\nu_{j}}\left(V_{j}^{n}-\max \left(V_{j}^{n}, V_{j-1}^{n}\right)\right), \\
B_{j}^{+}:=\min \left(V_{j}^{n}, V_{j-1}^{n}\right)+\frac{1}{\nu_{j}}\left(V_{j}^{n}-\min \left(V_{j}^{n}, V_{j-1}^{n}\right)\right),
\end{array}\right. \\
& \text { if } \nu_{j}<0, \quad\left\{\begin{array}{l}
b_{j}^{-}:=\max \left(V_{j}^{n}, V_{j+1}^{n}\right)+\frac{1}{\left|\nu_{j}\right|}\left(V_{j}^{n}-\max \left(V_{j}^{n}, V_{j+1}^{n}\right)\right), \\
B_{j}^{-}:=\min \left(V_{j}^{n}, V_{j+1}^{n}\right)+\frac{1}{\left|\nu_{j}\right|}\left(V_{j}^{n}-\min \left(V_{j}^{n}, V_{j+1}^{n}\right)\right) .
\end{array}\right.
\end{aligned}
$$

These numbers satisfy $b_{j}^{+} \leq B_{j}^{+}$, and $b_{j}^{-} \leq B_{j}^{-}$. There are useful in order to obtain stability properties. For instance, denoting $m_{j}:=\min \left(V_{j-1}^{n}, V_{j}^{n}\right)$ and $M_{j}=\max \left(V_{j-1}^{n}, V_{j}^{n}\right)$, we have as in [13] the following property:

$$
\left.\left.\left(\nu_{j} \in\right] 0,1\right], V_{j+\frac{1}{2}}^{n, L} \in\left[b_{j}^{+}, B_{j}^{+}\right], V_{j-\frac{1}{2}}^{n, R} \in\left[m_{j}, M_{j}\right]\right) \quad \Rightarrow \quad V_{j}^{n+1} \in\left[m_{j}, M_{j}\right]
$$

Now, we define the "fluxes" $V_{j+\frac{1}{2}}^{n, L}$ and $V_{j+\frac{1}{2}}^{n, R}$ as follows (see [7])

- If $\nu_{j}>0$ then define $V_{j+1 / 2}^{n, L}:=\min \left(\max \left(V_{j+1}^{n}, b_{j}^{+}\right), B_{j}^{+}\right)$;

- If $\nu_{j}<0$ then define $V_{j-1 / 2}^{n, R}:=\min \left(\max \left(V_{j-1}^{n}, b_{j}^{-}\right), B_{j}^{-}\right)$.

- If $\nu_{j} \leq 0$ and $\nu_{j+1} \geq 0$, then define

$$
V_{j+\frac{1}{2}}^{n, R}:=V_{j+1}^{n} \quad \text { and } \quad V_{j+\frac{1}{2}}^{n, L}:=V_{j}^{n} .
$$

- If $\nu_{j} \nu_{j+1}>0$, then define $V_{j+\frac{1}{2}}^{n, R}:=V_{j+\frac{1}{2}}^{n, L}\left(\right.$ if $\left.\nu_{j}>0\right)$ or $V_{j+\frac{1}{2}}^{n, L}:=V_{j+\frac{1}{2}}^{n, R}\left(\right.$ if $\left.\nu_{j+1}<0\right)$.

Note that in the case $\nu_{j}>0 \forall j$, we have $V_{j+\frac{1}{2}}^{n, R}=V_{j+\frac{1}{2}}^{n, L}$ and thus, denoting $V_{j+\frac{1}{2}}^{n}=$ $V_{j+\frac{1}{2}}^{n, L}$, the scheme (22) takes the more simple form

$$
V_{j}^{n+1}=V_{j}^{n}-\nu_{j}\left(V_{j+\frac{1}{2}}^{n}-V_{j-\frac{1}{2}}^{n}\right) .
$$

Here we check easily that $V_{j+\frac{1}{2}}^{n}, V_{j-\frac{1}{2}}^{n}$ satisfies the assumptions of (25), hence we obtain the stability $\min \left(V_{j-1}^{n}, V_{j}^{n}\right) \leq V_{j}^{n+1} \leq \max \left(V_{j-1}^{n}, V_{j}^{n}\right)$. In the general case $\left|\nu_{j}\right| \leq 1$, we would obtain

$$
\min \left(V_{j-1}^{n}, V_{j}^{n}, V_{j+1}^{n}\right) \leq V_{j}^{n+1} \leq \max \left(V_{j-1}^{n}, V_{j}^{n}, V_{j+1}^{n}\right)
$$


For other stability and convergence properties of this scheme, we refer to [7].

We also introduce the notation $F^{L}$ and $F^{R}$ in the case $V^{n}$ is a one-dimensional vector:

$$
V_{i+\frac{1}{2}}^{n, L}=F^{L}\left(V^{n}\right)_{i+\frac{1}{2}}
$$

and

$$
V_{i-\frac{1}{2}}^{n, R}=F^{R}\left(V^{n}\right)_{i-\frac{1}{2}}
$$

UB scheme for $2 d$ linear advection. Now we consider the equation

$$
\begin{array}{r}
v_{t}+f_{1}\left(x_{1}, x_{2}\right) v_{x_{1}}+f_{2}\left(x_{1}, x_{2}\right) v_{x_{2}}=0, \\
v\left(0, x_{1}, x_{2}\right)=v_{0}\left(x_{1}, x_{2}\right),
\end{array}
$$

where $x=\left(x_{1}, x_{2}\right)$ belongs to a square box of $\mathbb{R}^{2}$. We consider a Cartesian mesh $\left(x_{1, i}, x_{2, j}\right)\left(i \in\left\{1, \ldots, P_{x_{1}}\right\}, j \in\left\{1, \ldots, P_{x_{2}}\right\}\right)$, with constant mesh sizes $x_{1, i+1}-x_{1, i}=$ $\Delta x_{1}$ and $x_{2, j+1}-x_{2, j}=\Delta x_{2}$, and assume the CFL condition

$$
\max _{i, j}\left(\max \left(\frac{\Delta t}{\Delta x_{1}}\left|f_{1}\left(x_{1, i}, x_{2, j}\right)\right|, \frac{\Delta t}{\Delta x_{2}}\left|f_{2}\left(x_{1, i}, x_{2, j}\right)\right|\right)\right) \leq 1 .
$$

The UB scheme (22) is extended to (27) as proposed in [16], i.e. by using simply a Trotter splitting (or "alternate direction method", see [12]) The initialization step is

$$
V_{i, j}^{0}=\frac{1}{\Delta x_{1} \Delta x_{2}} \int_{I_{i} \times J_{j}} v_{0}\left(x_{1}, x_{2}\right) d x_{1} d x_{2}
$$

where $I_{i}=\left[x_{1, i}-\frac{\Delta x_{1}}{2} ; x_{1, i}+\frac{\Delta x_{1}}{2}\right]$ and $J_{j}=\left[x_{2, j}-\frac{\Delta x_{2}}{2} ; x_{2, j}+\frac{\Delta x_{2}}{2}\right]$.

We first make an evolution in the $x_{1}$-direction using

$$
V_{i, j}^{n, 1}=V_{i, j}^{n}-\frac{\Delta t}{\Delta x_{1}} f_{1}\left(x_{1, i}, x_{2, j}\right)\left(F^{L}\left(V_{., j}^{n}\right)_{i+\frac{1}{2}}-F^{R}\left(V_{\cdot, j}^{n}\right)_{i-\frac{1}{2}}\right)
$$

where $V_{\cdot, j}^{n}=\left(V_{i, j}^{n}\right)_{i=1, \ldots, P_{x_{1}}}$. Then we evolve in the $x_{2}$-direction using

$$
V_{i, j}^{n+1}=V_{i, j}^{n, 1}-\frac{\Delta t}{\Delta x_{2}} f_{2}\left(x_{1, i}, x_{2, j}\right)\left(F^{L}\left(V_{i, .}^{n, 1}\right)_{j+\frac{1}{2}}-F^{R}\left(V_{i, .}^{n, 1}\right)_{j-\frac{1}{2}}\right)
$$

where $V_{i, .}^{n, 1}=\left(V_{i, j}^{n, 1}\right)_{j=1, \ldots, P_{x_{2}}}$.

The CFL condition (29) is natural because here we consider a Trotter splitting.

F. Lagoutière [16] proved the very interesting property that the UB scheme advects exactly a particular class of step functions, in the case of constant advection. For instance, for 2-dimensional problems, let $u_{0}$ such that $V_{i j}^{0}$ initialized as in (30) belongs to the following space $\mathcal{S}$ :

$$
\mathcal{S}:=\left\{\left(V_{i, j}\right), \forall(a, b) \in\{0,1,2\}, V_{3 i+a, 3 j+b}=V_{3 i, 3 j}\right\} .
$$


Consider the UB scheme for $v_{t}+f \cdot \nabla v=0$ where $f=\left(f_{1}, f_{2}\right)=$ const is a constant advection vector of $\mathbb{R}^{2}$. Then, assuming the CFL condition $\max \left(\left|f_{1}\right| \frac{\Delta t}{\Delta x_{1}},\left|f_{2}\right| \frac{\Delta t}{\Delta x_{2}}\right) \leq 1$, we have $\forall i, j$ and $n \geq 0$ :

$$
V_{i, j}^{n}=\frac{1}{\Delta x_{1} \Delta x_{2}} \int_{I_{i} \times J_{j}} v\left(t_{n}, x_{1}, x_{2}\right) d x_{1} d x_{2}
$$

where $v\left(t, x_{1}, x_{2}\right)=v_{0}\left(x_{1}-f_{1} t, x_{2}-f_{2} t\right)$ is the exact solution (see also [16] for more general functions that are exactly advected).

It is this exact transportation property, which corresponds to an "antidissipative" behavior of the UB scheme, which motivates us for using it in front propagation problems such as (10). We refer to Désprès and Lagoutière[13] for other interesting properties of the UB scheme.

HJB-UB scheme. We now consider the discretization of an HJB equation of the form:

$$
v_{t}-\min _{u \in U}(f(x, u) \cdot \nabla v)=0, \quad t>0, x \in K
$$

We assume the CFL condition (29). The initialization of $V_{i, j}^{0}$ is done as in (30). Let $N_{u} \geq 1$ be an integer and $\left(u_{k}\right)_{k=1, \ldots, N_{u}}$ be a given discretization of the admissible set $U$. At time $t=t_{n}$, we denote by $V_{i, j}^{n+1, U B}(u)$ the UB scheme obtained from $\left(V_{i, j}^{n}\right)$ by using the advection $f(\cdot, u)$ with control $u$, i.e., one time step of the UB scheme for

$$
v_{t}-f(x, u) \cdot \nabla v=0 .
$$

Then the HJB scheme is given by

$$
V_{i, j}^{n+1}=\min _{u_{k}}\left(V_{i, j}^{n+1, U B}\left(u_{k}\right)\right) .
$$

We refer to [7] for first applications of the "Ultra-Bee" scheme to the resolution of HJB equations with discontinuous initial data. The scheme seems well adapted to treat discontinuous solutions and in particular when the value function takes only two values (0 and 1). Presently we have convergence results for the HJB-UB scheme for (31) in one space dimension but not in two dimensions.

$H J B-U B$ scheme for the computation of a capture basin before time $T$

The algorithm for computing a capture basin $\operatorname{Capt}_{F}(C ; T)$ before time $T$, for a given target $C$, is the following in the $2 \mathrm{~d}$ setting. Here we assume that $x \in \mathbb{R}^{2}$. Our aim is to discretize, for a given $T>0$, the function

$$
V(t, x):=\vartheta_{T}(T-t, x)
$$

where $\vartheta_{T}$ obeys equations (18). 
Let $\rho:=\left(\Delta x_{1}^{2}+\Delta x_{2}^{2}\right)^{1 / 2}\left(\Delta x_{1}\right.$ and $\Delta x_{2}$ are the mesh sizes $)$, and $C_{\rho}:=\{x \in$ $C, d(x, \partial C)>\rho\}$, where $d$ is the Euclidean distance $\left(C_{\rho}\right.$ is a subset of $\left.C\right)$. Then we consider for $u \in U$ and $v \in[0,1]$ the following approximation $\mathcal{W}_{\rho}$ of $\mathcal{W}$ (see (9)):

$$
\mathcal{W}_{\rho}(z)= \begin{cases}\{1\} & \text { if } z \notin C, \\ {[0,1]} & \text { if } z \in C \text { and } z \notin C_{\rho}, \\ \{0\} & \text { if } z \in C_{\rho} .\end{cases}
$$

Note that for $\rho=0$ we recover $C_{0}=\operatorname{int}(C)$ and $\mathcal{W}_{0}=\mathcal{W}$.

In this way, we enlarge the behavior of the dynamics near $\partial C$, so that a discretization scheme will be able to detect a change near $\partial C$.

Finally the scheme we propose is the HJB-UB scheme applied to the following HJB equation:

$$
v_{t}-\min _{u_{k} \in U, w \in \mathcal{W}_{\rho}(x)}\left(w f\left(x, u_{k}\right) \cdot \nabla v\right)=0,
$$

where $\left(u_{k}\right)_{k=1, \ldots, N}$ is a given discretization of the set $U$. Equivalently, we have

$$
V_{i, j}^{n+1}=\min _{u_{k}, w \in \mathcal{W}_{\rho}\left(x_{i, j}\right)}\left(V_{i, j}^{n+1, U B}\left(u_{k}, w\right)\right),
$$

where $V_{i, j}^{n+1, U B}\left(u_{k}, w\right)$ denotes the value computed with the associated dynamics $w$. $f\left(x_{i, j}, u_{k}\right)$ on the cell containing $x_{i, j}$.

Stopping criteria. For the computation of a viability kernel or a capture basin using the UB scheme, the principle is first to evolve in time and compute some approximation of $V(t, x)$ using the HJB-UB algorithm, (using a time step $\Delta t>0$ satisfying the CFL condition (29)). Then we decide to stop the scheme when the values $V_{i, j}^{n}$ are numerically converging. This means in particular that the capture basin $\operatorname{Capt}_{F}(C)$ is approximated by $\operatorname{Capt}_{F}(C ; T)$ for some $T>0$. In practice, for the first two tests of the following section, the UB scheme is stopped when the quantity $\left\|V^{n}-V^{n-1}\right\|_{L^{1}}:=$ $\Delta x_{1} \Delta x_{2} \sum_{i, j}\left|V_{i, j}^{n}-V_{i, j}^{n-1}\right|$ satisfies:

$$
\left\|V^{n}-V^{n-1}\right\|_{L^{1}} \leq 10^{-4}
$$

\section{$4 \quad$ Numerical tests}

In the following numerical tests, for the viability algorithm, we have used the basic version as presented in [20].

For practical purpose, the $+\infty$ value can be replaced by +1 , and in particular the initial (resp. border) condition $V(0, x)=+\infty$ (resp. $V(t, x)=+\infty)$ can be replaced by $V(0, x)=+1(\operatorname{resp} . V(t, x)=+1)$. 
Example 1 (consumption problem) We consider the problem of computing the viability kernel for:

$$
\begin{aligned}
& \dot{y}_{1}(t)=y_{1}(t)-y_{2}(t), \\
& \dot{y}_{2}(t)=u
\end{aligned}
$$

with a control $u \in U:=\left[-\frac{1}{2}, \frac{1}{2}\right]$, and the constraints $y_{1}(t) \in[0,2]$ and $y_{2}(t) \in[0,3]$. This corresponds to a consumption problem [20,3]. Hence, here $K:=[0,2] \times[0,3]$ and the corresponding HJB equation is

$$
\begin{aligned}
& V_{t}+\max _{u= \pm \frac{1}{2}}\left(-f\left(x_{1}, x_{2}, u\right) \cdot \nabla v\right)=0, \quad \forall t>0, \forall\left(x_{1}, x_{2}\right) \in K, \\
& V\left(0, x_{1}, x_{2}\right)=0, \quad \forall\left(x_{1}, x_{2}\right) \in K,
\end{aligned}
$$

where $f\left(x_{1}, x_{2}, u\right)=\left(\begin{array}{c}x_{1}-x_{2} \\ u\end{array}\right)$, and $V\left(t, x_{1}, x_{2}\right)=1, \quad \forall\left(x_{1}, x_{2}\right) \notin K, t \geq 0$. We have replaced the $+\infty$ value by 1 for commodity.

We have plotted in Fig. 1 the results given by the viability algorithm and by the HJB-UB scheme, for various mesh size $\left(P_{x_{1}}=P_{x_{2}}=50\right.$ and 100). For the UB scheme we have used time steps $\Delta t \simeq 0.013$ and 0.007 respectively, and stopped the computation at time $t_{n}=n \Delta t=5$ approximately. We have also used $N_{u}=2(u \in$ $\left.\left\{-\frac{1}{2}, \frac{1}{2}\right\}\right)$. The black lines delimit the border of the exact solution.

Note that the viability algorithm computes values 0 or 1 . In our algorithm, we compute values which are 0 or 1 , or some intermediary value. The intermediary values are observed to be always on a "frontier" which bandwidth is about one or two mesh size. The error on this frontier is not diffused by the scheme (to the contrary to most numerical methods as Semi-Lagrangian or finite difference methods), but stays well localised in a small bandwidth.

In Fig. 1 and the following, the small black square regions represent the computed viability kernel (or capture basin).

For the UB scheme, the black square regions are associated with the points where $0 \leq V_{i j}^{n} \leq \epsilon$ with $\epsilon=10^{-10}$ (the points from which we should be able to reach the target in time lesser than or equal to $t_{n}$ ); the grey points represents the mesh box with an intermediary value of $V_{i, j}^{n}$ between 0 (black) and 1 (white). More precisely, these boxes are represented if $\epsilon \leq V_{i, j}^{n} \leq 1-\epsilon$. This correspond to mesh boxes where the discontinuity is detected.

Example 2 (Zermelo problem). In this example, we compute the capture basin for a "Zermelo Problem":

$$
\begin{aligned}
& \dot{y}_{1}(t)=1-a y_{2}(t)^{2}+u \cos (\theta), \\
& \dot{y}_{2}(t)=u \sin (\theta)
\end{aligned}
$$

in the domain $\left(y_{1}, y_{2}\right) \in K:=[-6,2] \times[-2,2]$, and for controls $0 \leq u \leq u_{\max }:=0.44$, $\theta \in[0,2 \pi$ [, with $a=0.1$. The target is chosen here to be the ball $C:=B(0, r)$ with $r=0.44$. 

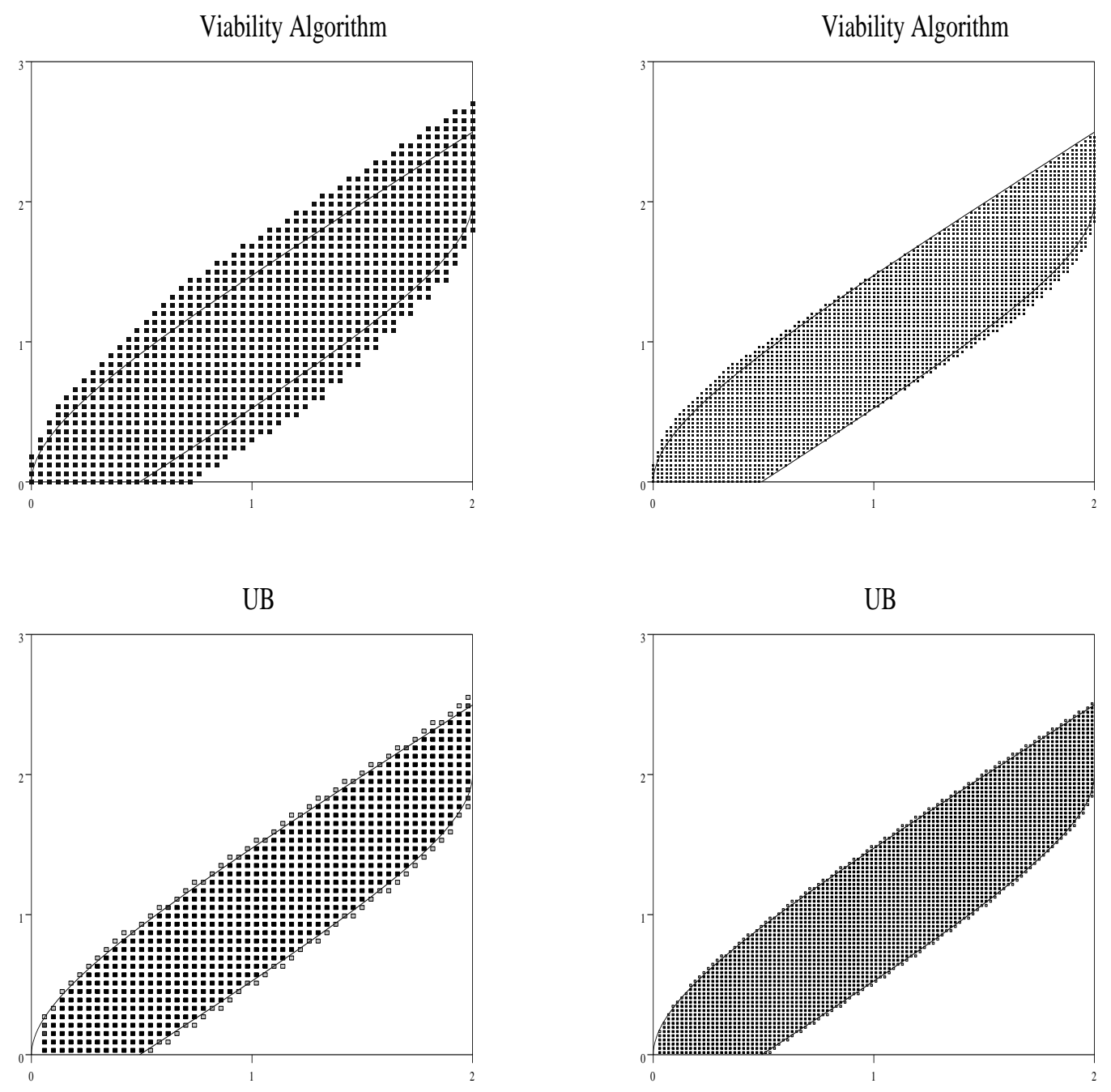

Figure 1: Comparison of the viability algorithm and the UB scheme for the consumption problem (left: $P_{x_{1}}=P_{x_{2}}=50$, right: $P_{x_{1}}=P_{x_{2}}=100$ ). 
The viability algorithm (see [22] in this case) and the UB scheme are compared in Fig.2, with $P_{x_{1}}=P_{x_{2}}=100$. For the UB scheme, we have used $N_{u}=20$ points and $d t \simeq 0.019$. the stopping criteria was $\left\|V^{n}-V^{n-1}\right\|_{L^{1}} \leq 10^{-4}$, which gave a stopping time $t \simeq 7$.

The circle delimits the border of the target, and the black lines also delimit the exact capture basin (we have computed the limit trajectories by using the Pontryagin Principle, see Bryson and Ho [9]).

Viability Algorithm

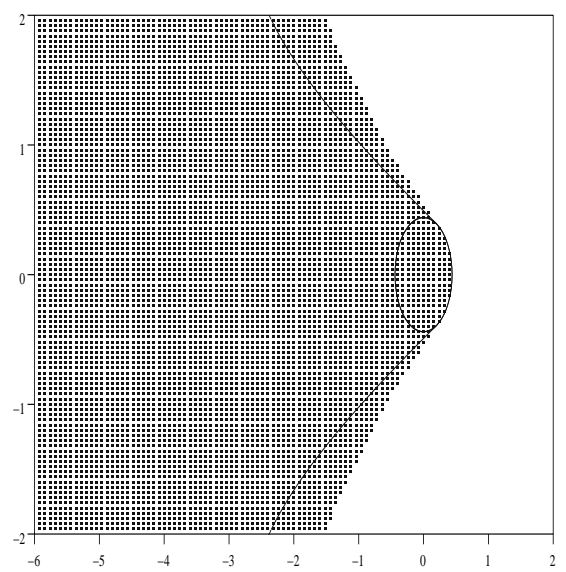

UB

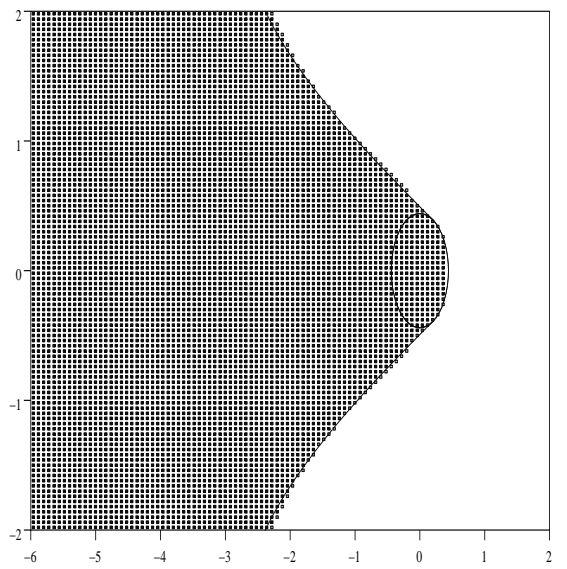

Figure 2: Approximation of the capture basin for the zermelo problem, $P_{x_{1}}=P_{x_{2}}=100$

Note that a good preliminary approximation is also obtained by the UB scheme even with a small number of mesh points. For instance in Fig. 3 we have used $P_{x_{1}}=P_{x_{2}}=20$ (with same number of controls, and $\Delta t \simeq 0.27$ ).

Example 3. In this example, we compute the "capture basin" for the following $2 \mathrm{~d}$ rotational dynamic:

$$
f\left(x_{1}, x_{2}, u\right)=\left(\begin{array}{r}
x_{2} \\
-x_{1}
\end{array}\right)
$$

on the domain $K=[-1,1]^{2}$. The target is the ball centered in $(0.5,0)$ and of radius 0.2 , i.e.,

$$
C:=\left\{\left(x_{1}, x_{2}\right) \in K,\left(x_{1}-0.5\right)^{2}+x_{2}^{2} \leq 0.2^{2}\right\} .
$$

Note that in the dynamic $f$ there is no dependency over a control $u$; however, the UB scheme does use a dynamic that depends of a control $w$ as in (35) (hence in practice we have $N_{u}=2$ ).

In Fig. 4 we compare the viability algorithm and the UB scheme at time $T=\pi$ (half a turn), with $P_{x_{1}}=P_{x_{2}}=100$ (and $\left.\Delta t=0.02\right)$ and $P_{x_{1}}=P_{x_{2}}=200($ and $\Delta t=0.01)$. 


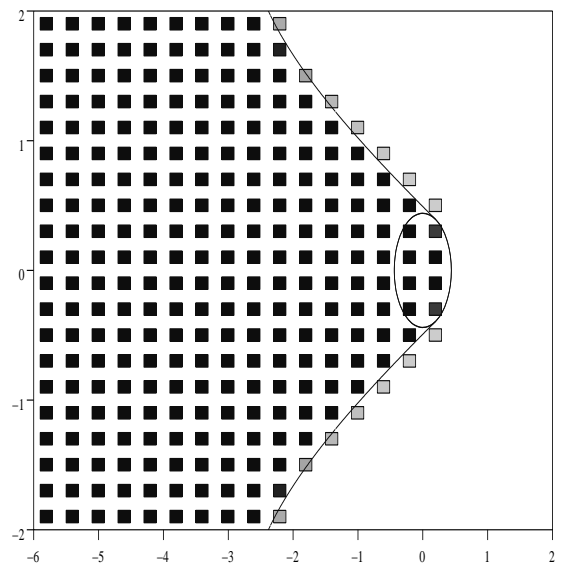

Figure 3: Zermelo problem, $P_{x_{1}}=P_{x_{2}}=20$

The small circle delimits the target and the black line reprensents the border of the exact solution.

In Fig. 5 we show the results with the UB scheme at time $T=10 \pi$ (five turns), with $P_{x_{1}}=P_{x_{2}}=50$ and $P_{x_{1}}=P_{x_{2}}=100(\Delta t=0.04$ and $\Delta t=0.02$ respectively $)$. We see that the UB scheme present no visible diffusion, even on a long time period, whereas the viability algorithm - not shown for the case $T=10 \pi$ - has a tendency to diffuse more and more with time.

This example well illustrates the problem of diffusion of some schemes. A diffusive algorithm is going to create more and more errors as we go far from the target (or as time goes on). However the anti-diffusive scheme well approximates the capture basin even for long time as illustrated in Fig.5. (We have used $P_{x_{1}}=P_{x_{2}}=25,50$ and 100 with $\Delta t=0.077,0.038$ and 0.019 resp.)

Example 4. In this example we compute the capture basin for the following target problem

$$
f\left(x_{1}, x_{2}, u\right)=\left(\begin{array}{l}
x_{2} \\
u
\end{array}\right)
$$

on a domain $K=[-1,1]^{2}$, with control $u \in[-1,1]$ (note that the dynamic also satisfies $\left.\ddot{x}_{1}=u\right)$. The target is a "thin target" $C:=\{(0,0)\}$. Numerically, the mesh for the UB scheme is chosen so that $(0,0)$ be the center of a mesh box of size $\left(\Delta x_{1}, \Delta x_{2}\right)$, and the initial data is $V_{i, j}=0$ if $\left(x_{1, i}, x_{2, j}\right)=(0,0)$ and $V_{i, j}=1$ otherwise (this corresponds to take $\left.v_{0}\left(x_{1}, x_{2}\right)=1_{\left\{\left|x_{1}\right| \leq \frac{\Delta x_{1}}{2},\left|x_{2}\right| \leq \frac{\Delta x_{2}}{2}\right\}}\right)$. Here the problem is discretised with three controls $u \in\{-1,0,1\}$.

The results are given in Fig.6. As before, we obtain a much smaller error and a smaller diffusion with the UB scheme than with the viability algorithm. 
Viability Algorithm

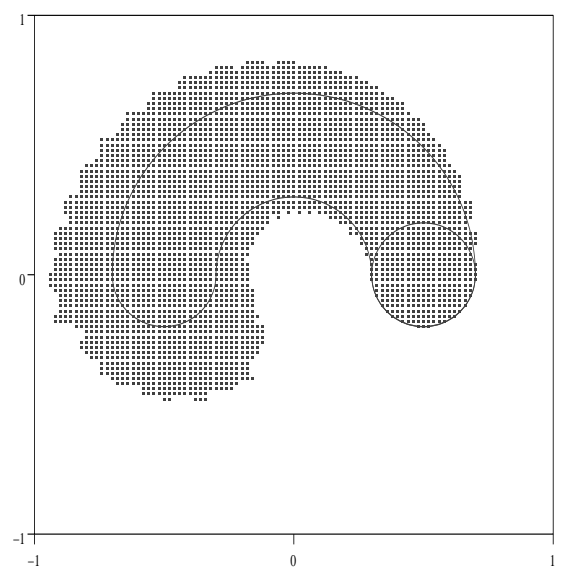

UB

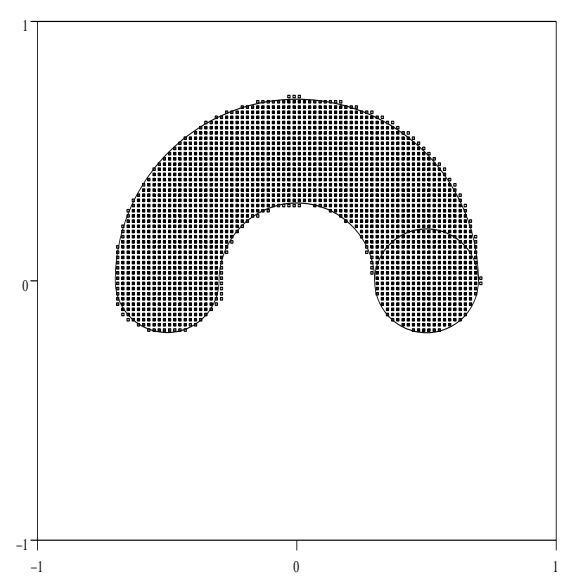

$P_{x_{1}}=P_{x_{2}}=100$
Viability Algorithm

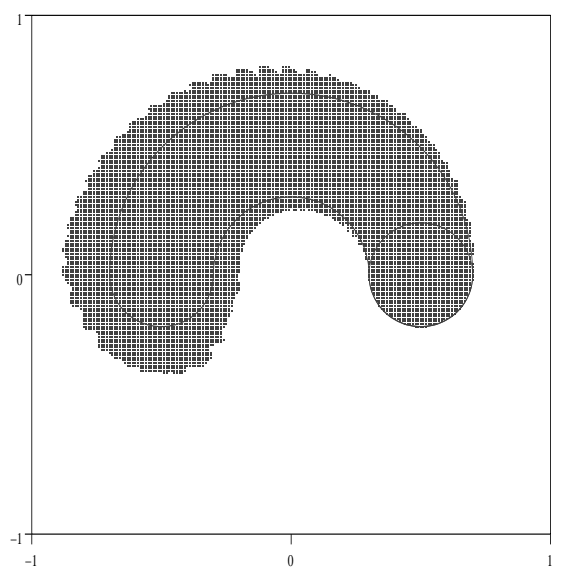

UB

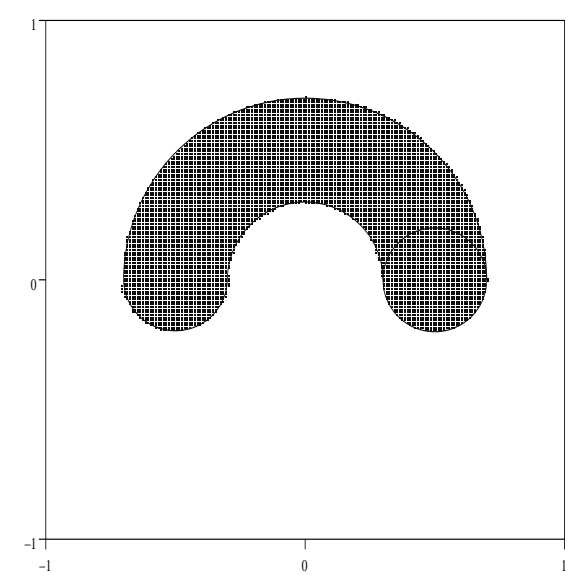

$P_{x_{1}}=P_{x_{2}}=200$

Figure 4: points that can reach the target in time $t \leq \pi$ 
$\mathrm{UB}$

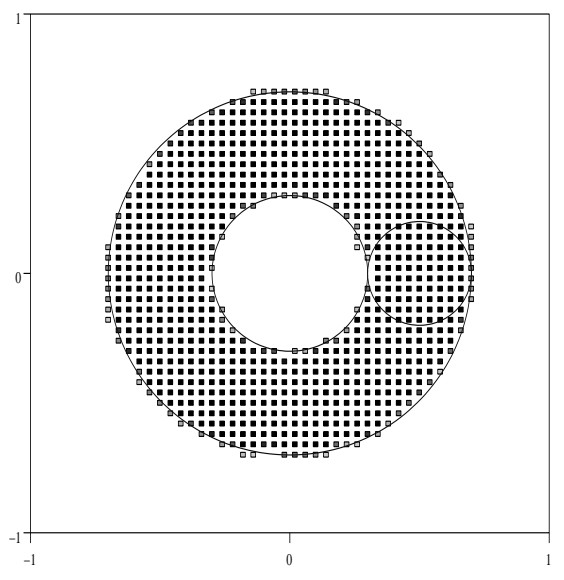

$P_{x_{1}}=P_{x_{2}}=50$
UB

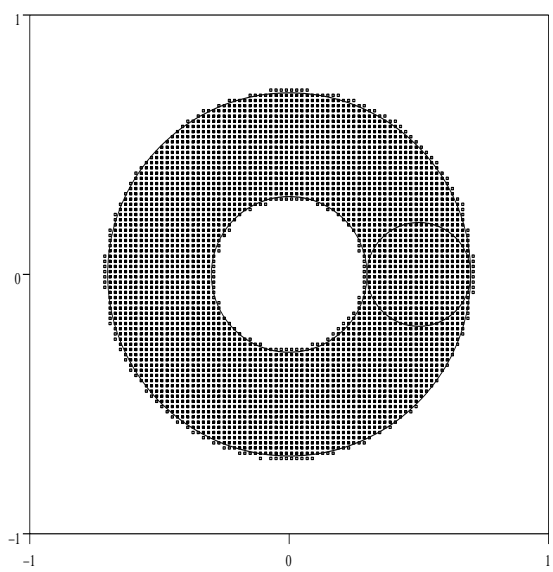

$P_{x_{1}}=P_{x_{2}}=100$

Figure 5: points that can reach the target in time $t \leq 10 \pi$ (UB scheme)

Acknowledgments. The authors wish to thank again P. Saint Pierre for useful discussions and for help using his code. We also thank the referees for useful remarks.

\section{A The viability Algorithm}

The approach followed by Saint-Pierre [20] is to determine the viability kernel in a constructive way by using discrete approximation. Also, Quincampoix and Saint-Pierre studied the case of a Hölderian differential inclusion [19]. In this case, the kernel is approximated by kernels of discrete dynamical systems and then by finite kernels of finite discrete dynamical systems.

Consider a compact subset $K \subset \mathbb{R}^{n}$, and the differential inclusion:

$$
y^{\prime}(t) \in F(y(t)) \text { a.e } t \geq 0, \quad y(0)=x_{0} \in K,
$$

where $F$ is upper-semi continuous set-valued map, with non empty convex compact values, and such that $\sup \|y\| \leq c(1+\|x\|)$ for some $c \geq 0$. With this inclusion, for a fixed $\rho>0$, we associate the discrete explicit scheme:

$$
\frac{y^{n+1}-y^{n}}{\rho} \in F\left(y^{n}\right) \quad \text { for } n \geq 0, \quad y^{0}=x_{0} \in K
$$

We denote by $G_{\rho}$ the set-valued map $G_{\rho}=1+\rho F$ and the system (39) can be rewritten as follows:

$$
y^{n+1} \in G_{\rho}\left(y^{n}\right) \text { for all } n \geq 0 .
$$

As far as the discrete dynamical system associated with $G$ is concerned, we denote the discrete viability kernel of $K$ under $G$ by $\operatorname{Viab}_{G}(K)$. 
Viability Algorithm

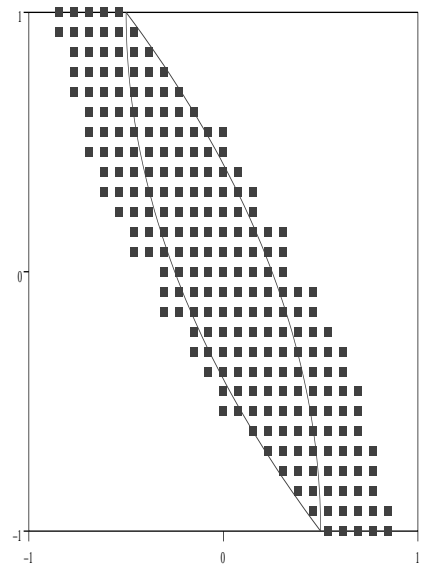

$\mathrm{UB}$

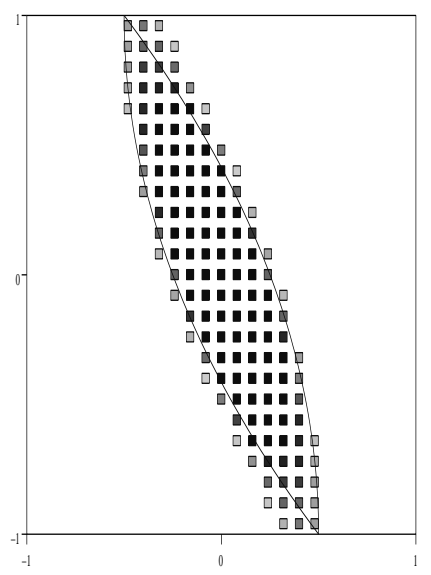

$P_{x_{1}}=P_{x_{2}}=26$
Viability Algorithm

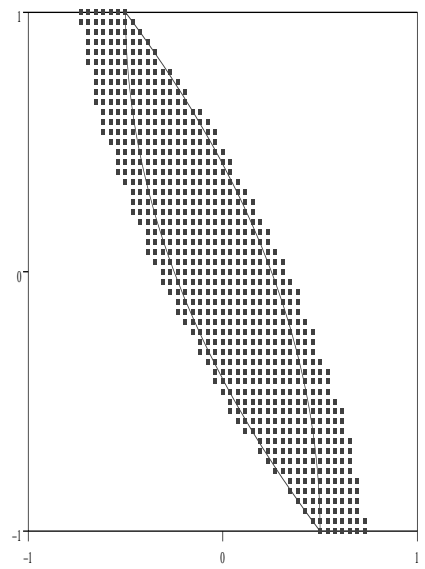

$\mathrm{UB}$

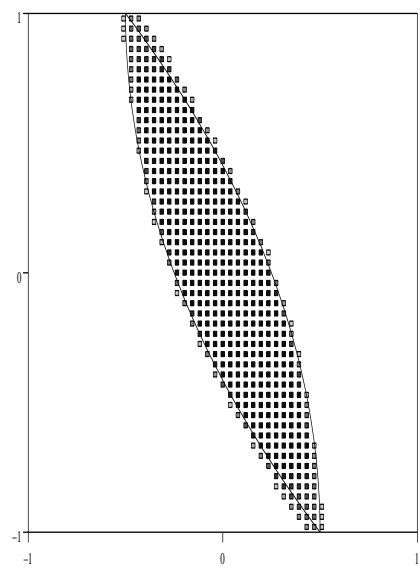

$P_{x_{1}}=P_{x_{2}}=52$
Viability Algorithm

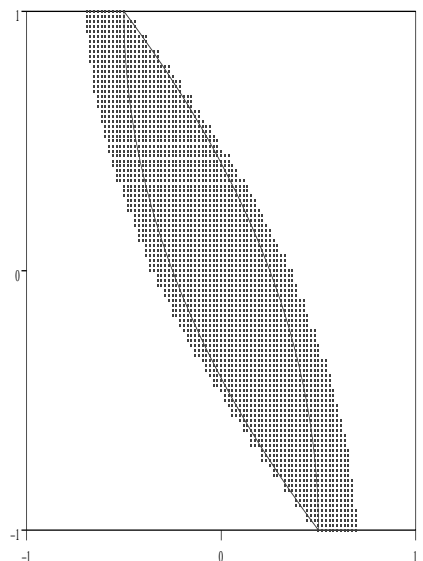

UB

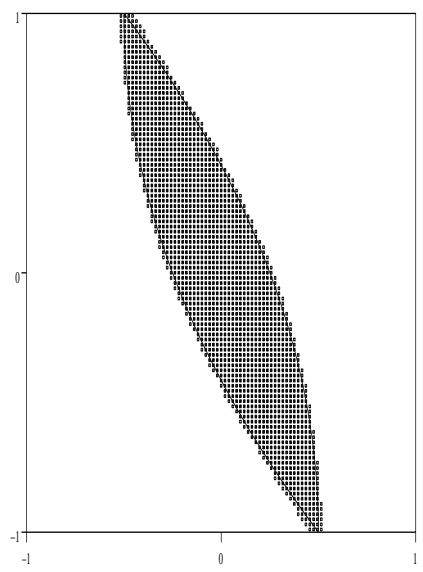

$P_{x_{1}}=P_{x_{2}}=104$

Figure 6: Cible problem, points that can reach the target in time $t \leq 1$ 


\section{A.1 Approximation by Kernels of Discrete Dynamical Sys- tems}

Saint-Pierre [20] first addresses the problem of the approximation of kernels of discrete dynamical systems. Under some assumptions, Saint-Pierre [20] proves that, if the sequence $\left(K^{n}\right)_{n}$ (with $K^{0}=K$ ) is defined as follows:

$$
K^{n+1}:=\left\{x \in K^{n} \text { such that: } G(x) \bigcap K^{n} \neq \emptyset\right\}
$$

then $\operatorname{Viab}_{G}(K)=\bigcap_{n=0}^{+\infty} K^{n}$. He next proves the convergence of the following approximation process:

Theorem A.1. We suppose furthermore that $F$ is an $\ell$-Lipschitz set-valued map (i.e., there exists $\left.\ell \geq 0, \forall y \in F(x), y^{\prime} \in F\left(x^{\prime}\right), d\left(y, y^{\prime}\right) \leq \ell d\left(x, x^{\prime}\right)\right)$.

Set $M:=\sup _{x \in K} \sup _{y \in F(x)}\|y\|$, and consider $F_{\rho}:=F+\frac{M \ell}{2} \rho B$ and $\Gamma_{\rho}:=1+\rho F_{\rho}$. Then

$$
\lim _{\rho \rightarrow 0} \operatorname{Viab}_{\Gamma_{\rho}}(K)=\operatorname{Viab}_{F}(K) .
$$

\section{A.2 Approximation by Finite Set-Valued Maps}

With any $h \in R$ we associate $X_{h}$ a countable subset of $\mathbb{R}^{n}$ for instance a grid with step $h$. Let $G_{h}: X_{h} \rightarrow X_{h}$ a finite set-valued map and a subset $K_{h} \subset \operatorname{Dom}\left(G_{h}\right)$. The finite dynamical system associated with $G_{h}$ is

$$
y_{h}^{n+1} \in G_{h}\left(y_{h}^{n}\right) \quad \forall n \geq 0 .
$$

Saint-Pierre remarks that, if the sequence $\left(K_{h}^{n}\right)_{n}$ (with $\left.K_{h}^{0}=K_{h}\right)$ is defined as by:

$$
K_{h}^{n+1}:=\left\{x \in K_{h}^{n} \text { such that: } G_{h}(x) \bigcap K_{h}^{n} \neq \emptyset\right\}
$$

then $\operatorname{Viab}_{G_{h}}\left(K_{h}\right)=\bigcap_{n=0}^{+\infty} K_{h}^{n}$. Moreover, there exist $p$ finite such that $\operatorname{Viab}_{G_{h}}\left(K_{h}\right)=K_{h}^{p}$.

The following proposition links finite discrete viability kernels and discrete viability kernels:

Proposition A.1. Let $G: \mathbb{R}^{n} \rightarrow \mathbb{R}^{n}$ be an upper semicontinuous set-valued map with closed values, $K$ a closed subset of $\operatorname{Dom}(G):=\{x \in X, G(x) \neq \emptyset\}$. Let $r>0$ be such that for all $x \in \operatorname{Dom}\left(G^{r}\right) \cap X_{h}, G^{r}(x) \cap X_{h} \neq \emptyset$, where $G^{r}$ is defined by $G^{r}(x)=G(x)+r B$. Then

$$
\operatorname{Viab}_{G^{r}}\left(K_{h}\right) \subset \operatorname{Viab}_{G^{r}}(K) \bigcap X_{h}
$$

Furthermore, for a good choice of $r$, these sets coincide. 
Gathering the preceding results Saint-Pierre proves the following convergence properties of approximations of viability kernel of $K$ under $F$ with finite viability kernels computable in a finite number of steps:

$$
\limsup _{\rho, h \rightarrow 0} \operatorname{Viab}_{G_{\rho h}^{2 M \ell \rho^{2}}}\left(K_{h}^{M l \rho^{2}}\right)=\operatorname{Viab}_{F}(K)
$$

with $G_{\rho}^{2 M \ell \rho^{2}}, G_{\rho h}^{2 M \ell \rho^{2}}$, and $K_{h}^{M l \rho^{2}}$ defined as follows :

$$
\begin{aligned}
& G_{\rho}^{2 M \ell \rho^{2}}(x):=x+\rho F(x)+2 M \ell \rho^{2} \mathcal{B}, \quad x \in \mathbb{R}^{n} ; \\
& G_{\rho h}^{2 M \ell \rho^{2}}(x):=G_{\rho h}^{2 M \ell \rho^{2}} \cap X_{h}, \quad x \in X_{h} ; \\
& K_{h}^{M \ell \rho^{2}}:=\left(K+M \ell \rho^{2}\right) \cap X_{h} .
\end{aligned}
$$

This viability kernel algorithm allows to compute the exact discrete and finite viability kernel of the associated discrete problem defined on a finite grid.

\section{References}

[1] R. Abgrall. Numerical discretization of boundary conditions for first order Hamilton-Jacobi equations. SIAM J. Numer. Anal., 41(6):2233-2261 (electronic), 2003.

[2] J.-P. Aubin and Arrigo Cellina. Differential inclusions, volume 264 of Comprehensive studies in mathematics. Springer, Berlin, Heidelberg, New York, Tokyo, 1984.

[3] J.P. Aubin. Viability theory. Birkhäuser, 1991.

[4] E.N. Barron and R. Jensen. Semicontinuous viscosity solutions for 'h'amilton'j'acobi equations with convex hamiltonians. Commun. in Partial Diff. Equations, 15(12):1713-1742, 1990.

[5] E.N. Barron and R. Jensen. Optimal control and semicontinuous viscosity solutions. Proceedings of the A.M.S, 113(2):397-402, 1991.

[6] C. Béné, L. Doyen, and D. Gabay. A viability analysis for a bio-economic model. Ecological Economics, 36:385-396, 2001.

[7] O. Bokanowski and H. Zidani. Anti-dissipative schemes for advection and application to Hamilton-Jacobi-Bellman equations. To appear in J. Sci. Computing, 2005.

[8] N. Bonneuil. Making ecosystem models viable. Bulletin of Mathematical Biology, 65:1081-1094, 2003. 
[9] A.E. Bryson and Y.C. Ho. Applied optimal control. Hemisphere Publishing, NewYork, 1975.

[10] F. Camilli and M. Falcone. Approximation of optimal control problems with state constraints: estimates and applications. In Nonsmooth analysis and geometric methods in deterministic optimal control (Minneapolis, MN, 1993), volume 78 of IMA Vol. Math. Appl., pages 23-57. Springer, New York, 1996.

[11] I. Capuzzo-Dolcetta and P.-L. Lions. Hamilton-Jacobi equation with state constraints. Trans. Amer. Math. Soc., 318:643-683, 1990.

[12] R. Dautray and J.-L. Lions. Mathematical Analysis and Numerical Methods for Science and Technology, volume 5, Evolution problems I. Springer (2000).

[13] B. Després and F. Lagoutière. Contact discontinuity capturing schemes for linear advection and compressible gas dynamics. J. Sci. Comput., 16:479-524, 2001.

[14] H. Frankowska and S. Plaskacz. Semicontinuous solutions of Hamilton-JacobiBellman equations with degenerate state constraints. J. Math. Anal. Appl., 251(2):818-838, 2000.

[15] H. Ishii and S. Koike. A new formulation of state constraint problems for first-order pdes. Siam J. Control Optim., 34(2):554-571, 1996.

[16] F. Lagoutière. PhD thesis, University of Paris VI, Paris, 2000.

[17] S. Martin. The cost of restoration as a way of defining resilience: a viability approach applied to a model of lake eutrophication. Eology and Society, 9(2):URL: http://www.ecologyandsociety.org/vol8/iss2/art8.

[18] C. Mullon, P. Curry, and L. Shannon. Viability model of trophic interctions in marine ecosystems. Natural Ressource Modelling, 17:2758, 2004.

[19] M. Quincampoix and P. Saint-Pierre. An algorithm for viability kernels in hölderian case: approximation by discrete dynamical systems. Journal of Mathematical Systems, Estimation, and Control, 5:1-13, 1995.

[20] P. Saint-Pierre. Approximation of viability kernel. Appl. Math. Optim., 29:187209, 1994.

[21] P. Saint-Pierre. Approche ensembliste des systèmes dynamiques: regards qualitatifs et quantitatifs. Matapli, (66):93-100, 2001.

[22] P. Saint-Pierre. Viable capture basin for studying differential and hybrid games: application to finance. Int. Game Theory Rev., 6(1):109-136, 2004.

[23] H.M. Soner. Optimal control with state-space constraint. I. SIAM J. Control Optim., 24(3):552-561, 1986. 